\title{
Psychological distress in patients with metastatic cancer enrolling on phase I clinical trials
}

\author{
Alexandra Hunt ${ }^{1} \cdot$ Elizabeth Handorf $^{2} \cdot$ Matthew Blau $^{3} \cdot$ Yana Chertock $^{3} \cdot$ Carolyn Fang $^{4} \cdot$ Michael J Hall $^{3} \cdot$ Rishi Jain $^{1}$
}

Received: 30 October 2020 / Accepted: 27 February 2021 / Published online: 13 March 2021

(C) The Author(s), under exclusive licence to Springer Science+Business Media, LLC, part of Springer Nature 2021

\begin{abstract}
Purpose Psychological distress is common in patients with cancer and is associated with lower quality-of-life (QOL). Although distress among oncology outpatients undergoing standard therapy has been widely studied, few studies have evaluated distress among patients enrolling on Phase I therapeutic clinical trials. Thus, we aimed to characterize levels of distress and types of stressors in patients enrolling on Phase I clinical trials.

Methods Participants completed the National Comprehensive Cancer Network Distress Thermometer (NCCN DT) and Problem list and measures of anxiety and depression at the time of Phase I clinical trial initiation.

Results We enrolled 87 patients (95\% with metastatic/incurable disease) who were initiating a Phase I clinical trial. Analyses revealed a high prevalence of distress (51\%) and anxiety (28\%). There were significant correlations between overall distress and practical problems $(r=0.31, p=0.016)$, family problems $(r=0.35, p=0.006)$, and emotional problems $(r=0.64, p<0.001)$, but not physical problems $(r=0.17, p=0.206)$.

Conclusions Patients may be better prepared to manage physical stressors but not practical, emotional, or family stressors at the time of Phase I trial enrollment.

Implications for Cancer Survivors Phase I trial patients experience high levels of distress which may be due to the rigors of previous therapies therapy and related emotional and social stressors related to the poor prognosis of their advanced cancer diagnosis. Distress may go unidentified without screening which is not standard practice at the time of Phase I trial consideration. Future studies should evaluate strategies to routinely identify and intervene upon addressable stressors in patients participating in Phase I clinical trials.
\end{abstract}

Keywords Psychological distress $\cdot$ Phase I clinical trials $\cdot$ Anxiety

\section{Introduction}

Psychological distress can arise at any time throughout the cancer treatment continuum. Psychological distress is particularly common among patients with metastatic cancer, and large studies using the National Comprehensive Cancer Network Distress Thermometer (NCCN-DT) have shown that

Rishi Jain

rishi.jain@fccc.edu

1 Department of Hematology/Oncology, Fox Chase Cancer Center, Philadelphia, PA 19111, USA

2 Biostatistics, Fox Chase Cancer Center, Philadelphia, PA 19111, USA

3 Department of Clinical Genetics, Fox Chase Cancer Center, Philadelphia, PA 19111, USA

4 Cancer Prevention and Control, Fox Chase Cancer Center, Philadelphia, PA 19111, USA greater than $50 \%$ of patients with advanced cancer may have significant distress $[1,2]$. The NCCN-DT is a commonly used instrument to measure overall distress and specific types of stressors including physical, religious/spiritual, emotional, family, and practical [3]. Distress has been associated with a variety of adverse outcomes including depression, anxiety, increased likelihood of missed appointments, and even worsened prognosis $[4,5]$. Thus, early identification of distress is of utmost importance.

Assessment tools such as the NCCN-DT are easy for healthcare providers to administer and can facilitate referral to the appropriate psychosocial services such a psychiatry, social work, or chaplaincy in an effort alleviate distress. Unfortunately, despite the development of such tools, distress commonly goes unrecognized and therefore untreated [6]. Little is known about the sources, prevalence, and clinical impact of distress in patients enrolling onto Phase I clinical trials. The Phase I trial population is unique for a variety of reasons. These patients usually have metastatic cancer and 
may experience physical symptoms from side effects of prior therapies including surgery, radiation, and systemic therapies (e.g., chemotherapy, targeted therapies, or immunotherapy). These patients are also at high risk for emotional stressors (e.g., anxiety regarding their disease progression), family stressors (e.g., burden of patient's illness on their spouse or children), or even practical concerns (e.g., impact of disease and treatment on patient's financial status). The objectives of this study were to determine which stressors (practical, emotional, familial, or physical) are most strongly associated with distress in Phase I clinical trial participants.

\section{Methods}

This study was approved by Fox Chase Cancer Center's (FCCC) Institutional Review Board. Eligible patients were English speaking adults $\geq$ age 18 , had a diagnosis of cancer, and were initiating a Phase I clinical trial at FCCC. Baseline characteristics were extracted from the medical record. The NCCN-DT was used to screen for psychological distress in patients with cancer [3]. This tool first asks the patient to rate their overall distress from 0 to 10 and then answer 39 yes/no questions regarding specific stressors divided into the following problem categories: practical, emotional, spiritual/religious, family, and physical problems. The NCCN-DT and Problem List was used to measure psychological distress with a score of $\geq 4$ used to define distress [3]. The Hospital Anxiety and Depression Scale (HADS) was used to define anxiety (HADS-A score $\geq 8$ ) or depression (HADS-D score $\geq 8$ ). The assessments were conducted on the day of clinical trial initiation (cycle 1, day 1), and responses were self-reported by each subject. Pearson's product moment correlation tested the relationship between distress and HADS A and HADS D. Spearman's rank correlation tested the relationship between distress and the number of positive responses in the individual NCCN Problem List categories (practical, family, emotional, and physical). Associations between measures were determined using Chi-square analysis and Fisher's exact test. Statistical analyses were performed using Stata version 12.

\section{Results}

The study enrolled 87 Phase I participants between July 2016 and May 2017. Baseline characteristics are shown in Table 1. Within this cohort of subjects, 83/87 (95\%) of the patients had advanced, metastatic disease. There was an even balance of male and female participants (48\% and 52\%, respectively). The majority of patients (71\%) had an Eastern Cooperative Oncology Group (ECOG) performance status of 1. Patients were accrued from a variety of disease sites: Gastrointestinal (32\%), Hematology (15\%), Genitourinary (14\%),
Table 1 Patient baseline characteristics

\begin{tabular}{|c|c|}
\hline Characteristic & $N=87$ \\
\hline Mean age (range) & $60(28-85)$ \\
\hline \multicolumn{2}{|l|}{ Gender } \\
\hline Male & $48 \%$ \\
\hline Female & $52 \%$ \\
\hline \multicolumn{2}{|l|}{ Stage } \\
\hline Non-metastatic & $5 \%$ \\
\hline Metastatic & $95 \%$ \\
\hline \multicolumn{2}{|l|}{ Performance status } \\
\hline ECOG 0 & $28 \%$ \\
\hline ECOG 1 & $71 \%$ \\
\hline ECOG 2 & $1 \%$ \\
\hline \multicolumn{2}{|l|}{ Disease site } \\
\hline GI & $32 \%$ \\
\hline Heme & $15 \%$ \\
\hline GU & $14 \%$ \\
\hline GYN & $13 \%$ \\
\hline Thoracic & $9 \%$ \\
\hline Sarcoma & $7 \%$ \\
\hline Melanoma & $6 \%$ \\
\hline $\mathrm{H} \& \mathrm{~N}$ & $2 \%$ \\
\hline Other & $2 \%$ \\
\hline \multicolumn{2}{|c|}{ No. of prior lines of therapy } \\
\hline $0-2$ & $56 \%$ \\
\hline$\geq 3$ & $44 \%$ \\
\hline \multicolumn{2}{|l|}{ Presence of distress } \\
\hline Yes $($ NCCN DT $\geq 4)$ & $51 \%$ \\
\hline No $(\mathrm{NCCN}$ DT < 4) & $49 \%$ \\
\hline \multicolumn{2}{|l|}{ Presence of anxiety } \\
\hline Yes (HADS-A $\geq 8$ ) & $28 \%$ \\
\hline No (HADS-A < 8) & $72 \%$ \\
\hline \multicolumn{2}{|l|}{ Presence of depression } \\
\hline Yes (HADS-D $\geq 8$ ) & $18 \%$ \\
\hline No (HADS-D < 8) & $82 \%$ \\
\hline
\end{tabular}

ECOG, Eastern Cooperative Oncology Group; NCCN DT, National Comprehensive Cancer Network Distress Thermometer

Gynecology (13\%), Thoracic (9\%), Sarcoma (7\%), Melanoma (6\%), Head and Neck (2\%), and Other (2\%).

The prevalence of psychological distress was $51 \%$. The prevalence of anxiety and depression was $28 \%$ and $18 \%$, respectively. The most common problems reported by NCCNDT Problem List category were as follows: practical (treatment decisions, financial issues, and work or school problems), family (partner issues, family health issues, or children problems), emotional (worry, nervousness, and fears), and physical (pain, sleep problems, and eating problems). Correlations between baseline distress and other measures are shown in Fig. 1. The number of emotional $(r=0.64, p<0.001)$, family $(r=0.35, p=$ $0.006)$, and practical stressors $(r=0.31, p=0.016)$ were positively correlated with higher levels of distress. However, the number of physical stressors was not correlated with overall distress $(r=0.17, p=0.206)$. There was a significant positive 
correlation between psychological distress and anxiety $(r=$ $0.47, p<0.001)$ but not depression $(r=0.20, p=0.062)$.

\section{Discussion}

Volunteers for Phase I clinical trials have often undergone surgery, radiation, and multiple systemic therapies prior to enrollment. Participation in a Phase I trial is often a final effort to fight a cancer that has become refractory to standard treatments. As expected, our study identified a high prevalence of psychological distress $(51 \%)$ in Phase I clinical trial participants, the majority of whom had metastatic disease and incurable, similar to other cancer
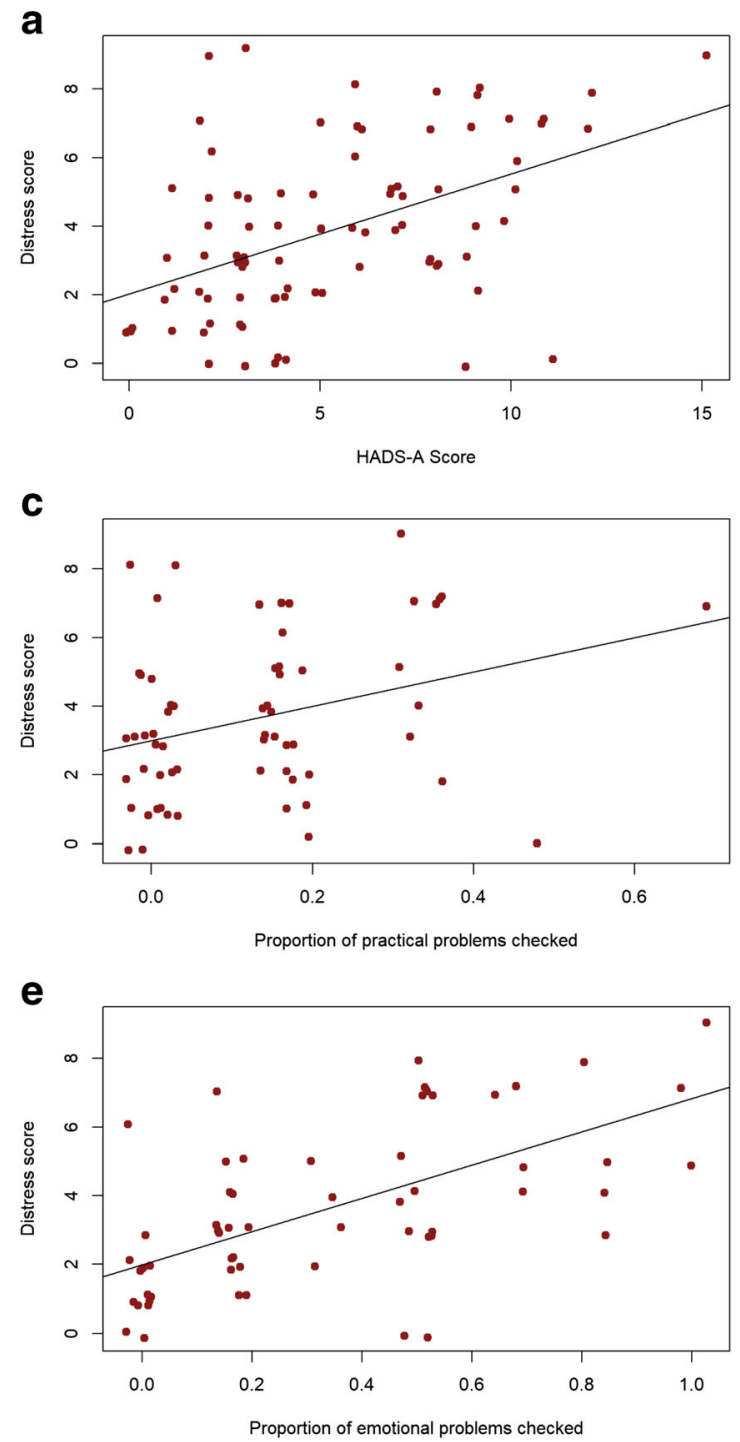

Fig. 1 a-f Correlations between distress, anxiety, depression, and specific stressors. a Correlation between distress and anxiety. b Correlation between distress and depression. c Correlation between populations $[1,2]$. Given the high rates, routine screening for distress in patients enrolling on Phase I clinical trials should be considered to ensure distress is systematically identified and addressed. To further elucidate the specific sources of distress in this population, we looked at the number of positive responses in four problem categories from the NCCN-DT Problem List in relation to overall distress. Interestingly, we found that emotional, family, and practical stressors were strongly correlated with overall distress, while physical stressors were not.

As patients enrolling on Phase I trials typically have advanced, incurable cancers and have usually been previously treated with one or more systemic cancer therapies, they are generally prepared for the physical stressors that accompany

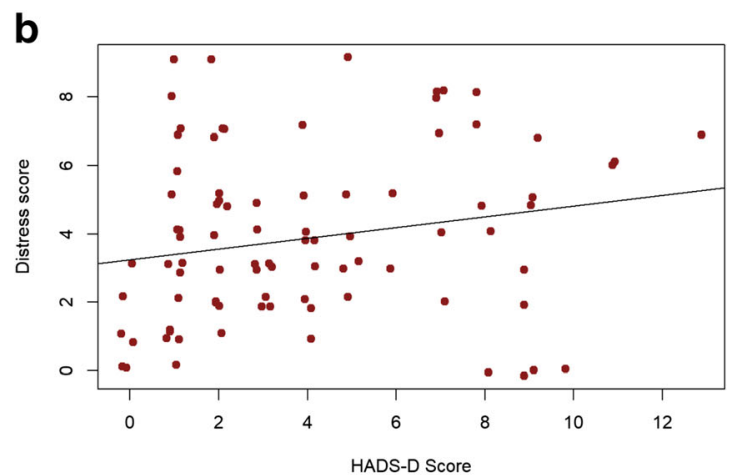

d

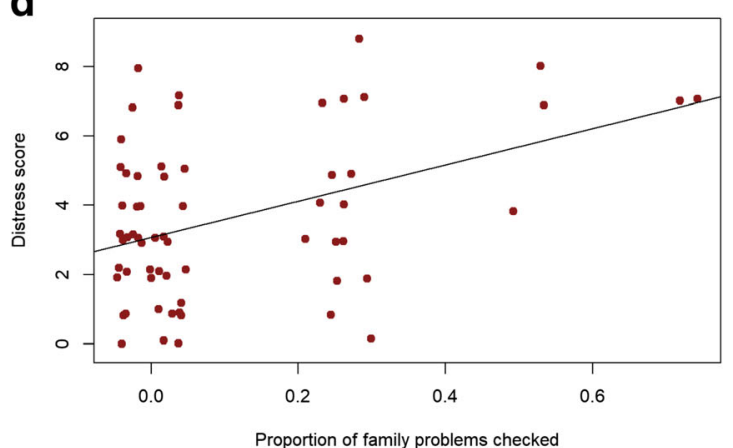

$\mathbf{f}$

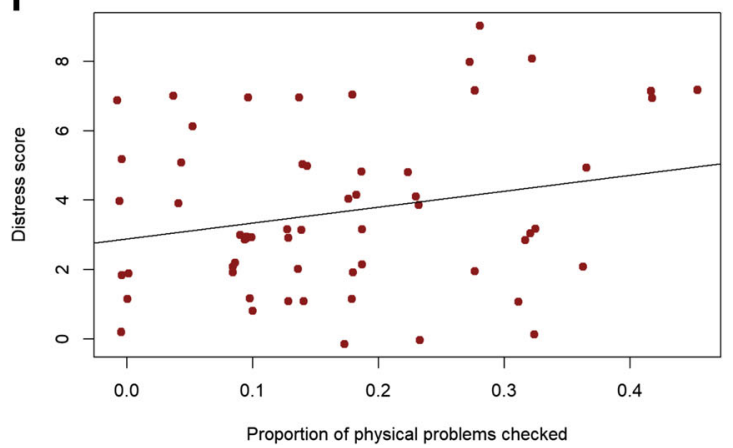

distress and practical problems. d Correlation between distress and family problems. e Correlation between distress and emotional problems. f Correlation between distress and physical problems 
their cancer diagnosis and associated treatments. At the time of initiation of any cancer therapy, oncologists spend a significant amount of time discussing potential risks of side effects that may arise from the respective therapy (e.g., nausea, mouth sores, or diarrhea). Providers also routinely reassess treatment side effects and symptom burden and each "toxicity check" after the initiation of systemic therapy to ensure any adverse effects are well controlled. Additionally, opioids are often used in conjunction with cancer treatment to manage pain occurring with the disease, which was one of the highest experienced physical problems in our population. These side effects or symptom inventories align closely with physical problems on the NCCN DT's Problem List [3]. We suspect that the lack of a correlation between baseline distress and physical stressors in our Phase I trial cohort largely arises from adequate preparation for these issues resulting from the significant amount of time oncology care teams dedicate to the physical problems that can arise throughout the cancer continuum.

While oncologists prepare patients for known symptom burden or treatment side effects, assessments of family, emotional, or practical (e.g., financial) concerns are not done as routinely in standard oncologic practice as are inventories of physical concerns and symptoms related to cancer treatment [6]. As shown in our population, patients facing an incurable cancer enrolling on a Phase I trial have a number of hardships at the time of trial consideration including financial, partner or child issues and may carry significant emotional burden with feelings of nervousness and worries likely related to potential risks and benefits of the experimental agents being studied. Unfortunately, these stressors may go unidentified at the time of study enrollment and distress may worsen as a result. Efforts to better support Phase I trial participants in all aspects of distress are in urgently needed as unmanaged emotional, family, and practical stressors may compromise QOL and could potentially be exacerbated by the rigors of clinical trial therapy. For instance, a practical problem such as a higher transportation burden due to more frequent clinical trial visits or an emotional problem with feelings of nervousness, fears, or worry about the risks and benefits of an experimental therapy may worsen due to participation on trial.

As therapeutic strategies for advanced cancer have become increasingly complex, a variety of barriers exist that limit patient access to psychosocial support. Patients may feel embarrassed when discussing family, practical, or emotional stressors which may lead to underdiagnoses. Access to social workers and clinical psychologists may also be limited leading to under treatment. Studies have demonstrated the feasibility of conducting interventions in the Phase I population [7, 8]. Smith et al. enrolled 479 patents onto a randomized trial evaluating an early palliative care intervention comprising of QOL assessments and interdisciplinary meetings to discuss care plans versus "usual care" and found that their intervention improved QOL and distress [7]. Treasure et al. enrolled 68 patients and found that those randomized to a structured palliative care intervention had increased duration on the Phase I trial, reduced adverse events, decreased symptom burden, and improved QOL [8]. Future studies should consider integrating support services (e.g., psychology, social work) from initial diagnosis specifically targeting emotional, family, and practical stressors in all Phase I trial participants to alleviate patient and family distress. While access to in-person supportive services may also be challenging due to the COVID-19 pandemic, remote psychological interventions which have been evaluated in other populations should be reconfigured for the Phase I trial population $[9,10]$. Ultimately, as in other areas in oncology, a multidisciplinary approach will be necessary to overcome the diverse variety of problems that can arise during trial therapy. For instance, if the trial leads to complications and hospitalization, practical issues (e.g. child care, financial, work) may predominate. However, if the trial therapy is unsuccessful, emotional distress would likely predominate and may necessitate support from all members of the team as part of a family meeting. Additional research should be done to evaluate collaborative models incorporating oncologists, psychology, social work, and palliative care specialists to help manage the unique causes of distress during Phase I trial therapy. Furthermore, as patients on Phase I trials often spend many hours with the nursing teams due to long infusion times, serial lab draws, and/or post-treatment toxicity monitoring, nurses should be included as a key source of support in collaboration with other team members.

There are limitations of our study. As we conducted a onetime assessment of distress at baseline, we do not know how distress changed longitudinally over the duration of the Phase I clinical trial which could inform when these participants would benefit most from screening and intervention. Our cohort was also quite heterogeneous including patients with a variety of cancers and treatments; thus, distress could differ from other Phase I cohorts at different centers. Nevertheless, our data is highly valuable as there is little data on the prevalence and clinical significance of psychological distress in the Phase I population.

In conclusion, our study found a high prevalence of psychological distress and strong correlations between practical, family and emotional stressors, and overall distress in Phase I trial participants. Future studies should identify strategies to systematically screen patients for these stressors and ensure patients are promptly triaged to the appropriate multidisciplinary support services.

\section{Declarations}

Conflict of interest The authors declare no conflict of interest. 


\section{References}

1. Zabora J, BrintzenhofeSzoc K, Curbow B, Hooker C, Piantadosi S. The prevalence of psychological distress by cancer site. Psychooncology. 2001;10(1):19-28. https://doi.org/10.1002/10991611(200101/02)10:1<19::aid-pon501>3.0.co;2-6.

2. Mehnert A, Hartung TJ, Friedrich M, Vehling S, Brähler E, Härter $\mathrm{M}$, et al. One in two cancer patients is significantly distressed: prevalence and indicators of distress. Psycho-oncology. 2018;27(1):75-82. https://doi.org/10.1002/pon.4464.

3. Riba MB, Donovan KA, Andersen B, Braun I, Breitbart WS, Brewer BW, et al. Distress management, Version 3.2019, NCCN Clinical practice guidelines in oncology. J Natl Compr Cancer Netw. 2019;17(10):1229-49. https://doi.org/10.6004/jnccn.2019. 0048 .

4. Ownby KK. Use of the distress thermometer in clinical practice. Journal of the advanced practitioner in oncology. 2019;10(2):1759.

5. Kim GM, Kim SJ, Song SK, Kim HR, Kang BD, Noh SH, et al. Prevalence and prognostic implications of psychological distress in patients with gastric cancer. BMC Cancer. 2017;17(1):283. https:// doi.org/10.1186/s12885-017-3260-2.
6. Fallowfield L, Ratcliffe D, Jenkins V, Saul J. Psychiatric morbidity and its recognition by doctors in patients with cancer. $\mathrm{Br} \mathrm{J}$ Cancer. 2001;84(8):1011-5. https://doi.org/10.1054/bjoc.2001.1724.

7. Thomas J, Smith VC, Hughes MT, Koczywas M, Azad NS, Knight $\mathrm{L}$, et al. A randomized trial of a palliative care intervention for patients on phase I studies. J Clin Oncol. 2020;38(15_suppl): 12001-1.

8. Michelle Elizabeth Treasure BJD, PingFu F, Cao S, Meropol NJ, Dowlati A. A randomized, controlled trial of structured palliative care versus standard supportive care for patients enrolled on phase I clinical trials. J Clin Oncol. 2020;38(15_suppl):3634-4.

9. Wang P, Yu T, Yang L. Web-based remote psychological intervention improves cancer treatment. Psychol Health Med. 2017;22(7): 879-87. https://doi.org/10.1080/13548506.2016.1247212.

10. Beatty L, Koczwara B, Wade T. Evaluating the efficacy of a selfguided Web-based CBT intervention for reducing cancer-distress: a randomised controlled trial. Supportive care in cancer : official journal of the Multinational Association of Supportive Care in Cancer. 2016;24(3):1043-51. https://doi.org/10.1007/s00520-0152867-6.

Publisher's note Springer Nature remains neutral with regard to jurisdictional claims in published maps and institutional affiliations. 